Z. klin. Chem. u. klin. Biochem.

8. Jg., S. 516-519, September 1970

\title{
Screening auf Cystinurie und Homocystinurie
}

\author{
Manuelle und balbautomatische quantitative Bestimmung von aliphatischen Verbindungen mit SH+Gruppen und S-S-Brïcken \\ im Urin
}

\author{
Von E. MöNCh und H. SIEMES ${ }^{1}$ ) \\ Aus der Kinderklinik (Kaiserin-Auguste-Victoria-Haus, Direktor: Professor Dr. A. Loeschke †) \\ der Freien Universität Berlin
}

(Eingegangen am 30. April 1970)

Es wird ein sowohl manuell als auch halbautomatisch durchführbares kolorimetrisches Verfahren zur quantitativen Bestimmung der SH-Gruppen- bzw. S-S-Brücken-haltigen Aminosäuren im Urin beschrieben. Es beruht auf der Reaktion. von aliphatischen Thiolen mit 5,5'-Dithio-bis-(2-nitrobenzoesäure) unter Freisetzung eines gefärbten aromatischen Thiols, dessen Extinktion gemessen wird. Als Bezugswert dient die Kreatininkonzentration des Urines. Die Methode wird angewendet als Suchtest auf Cystinurie und Homocystinurie, bei denen stark erhöhte Konzentrationen SH-Gruppen- bzw. S-S-Brücken-haltiger Aminosäuren gefunden werden. Weniger stark erhöhte Werte fanden sich bei generalisierten Hyperaminoacidurien infolge von Rachitiden, Nephrosen, Atemnotsyndromen, Dyspepsie, Ileus und Duodenalstenose.

\section{Screening for cystinuria and bomocystinuria}

The manuah and semiautomatic quantitative determination of urinary aliphatic compounds containing $S H$ groups or $S$-S bridges

A manual and semiautomatic method are described for the quantitative determination of urinary amino acids containing SH groups or S-S bridges.

The method is based on the reaction of aliphatic thiols with 5-5'-dithio-bis-(2-nitrobenzoicacid); this releases a coloured, aromatic thiol, whose extinction is measured. The urinary creatinine concentration serves as the reference value.

The method is used as a screening test for cystinuria and homocystinuria, which are characterised by considerably increased urinary concentrations of SH- or S-S-containing amino acids.

Increased but less marked values were found in generalised hyperaminoaciduria, resulting from rickets, nephrosis, respiratory distress syndrome, dyspepsia, ileus and duodenal stenosis.

Cystinurie und Homocystinurie gehören zu den angeborenen Aminosäuren-Stoffwechselstörungen, bei denen eine frühzeitige Behandlung Erfolg verspricht. Bei der Cystinurie verhindert eine Therapie die Hypercystinurie und damit die Cystin-Steinbildung (ungefähr 1\% aller Steine) in den ableitenden Harnwegen. Bei der Homocystinurie lassen sich die schweren Veränderungen mit geistiger Retardierung, Linsenektopie und epileptiformen Krämpfen verringern oder sogar vermeiden.

$\mathrm{Da}$ beide Krankheiten in ihren Frühstadien symptomarm bzw. symptomlos verlaufen, muß man systematisch nach ihnen suchen. Trotz der verschiedenen Ursachen beider Erkrankungen haben sie die vermehrte Ausscheidung von Aminosäuren mit SH-Gruppen oder S-S-Brücken gemeinsam. Auf Grund dieser Gemeinsamkeit lassen sich beide Erkrankungen mit gleichen Mitteln diagnostizieren.

\section{Material und Methoden \\ Material}

Untersucht wurden in einer Querschnitts-Untersuchung unserer Klinik unpräparierte Morgenurine von Kindern verschiedener Altersgruppen.

Metbode

Zurückgegriffen wurde auf eine von ExLMAN (1) angegebene kolorimetrische Bestimmung von SH-Gruppen in biologischen

1) Diese Veröffentlichung enthält einen Teil der Doktorarbeit von $\mathrm{H}$. SiEmes.
Flüssigkeiten. Das wasserlösliche aromatische Disulfid 5,5'Dithio-bis-(2-nitrobenzoesäure) [I] reagiert mit aliphatischen SHVerbindungen bei $\mathrm{pH} 7,0-8,0$, wobei pro Mol SH-Gruppen $1 \mathrm{Mol}$ 2-Nitro-5-thiobenzoesäure-Anion [II] entsteht. Dieses Anion ist intensiv orangegelb gefärbt.
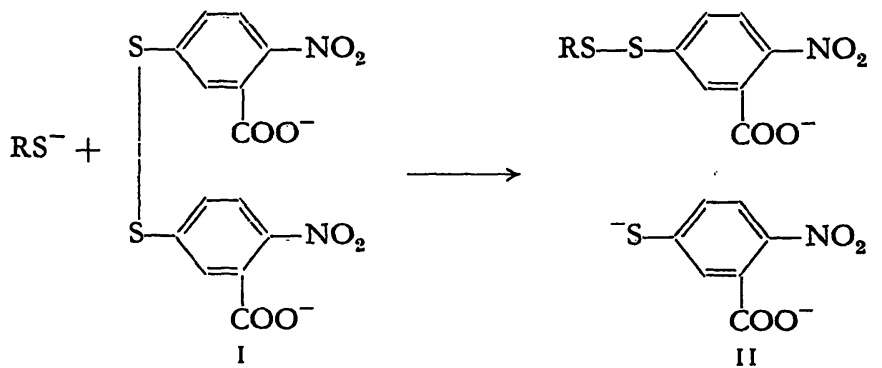

Im Urin kommen die SH-Gruppen-haltigen Aminosäuren in der oxydierten Form Cystin und Homocystin vor. Damit diese nach der Methode von Ellman bestimmt werden können, müssen zunächst Cystein bzw. Homocystein freigesetzt werden. Nach Sulrrvan und Mitarbeitern (2) benutzten wir dazu Natriumcyanid. Dieses reagiert nach der Gleichung

$$
\mathrm{R}-\mathrm{S}-\mathrm{S}-\mathrm{R}+\mathrm{NaCN} \rightleftharpoons \mathrm{R}-\mathrm{SNa}+\mathrm{R}-\mathrm{SCN},
$$

wobei pro Mol Cystin oder Homocystin $1 \mathrm{Mol}$ Cystein bzw. Homocystein freigesetzt wird.

\section{Bezugswert}

Die im Urin festgestellte SH-Gruppenkonzentration haben wir auf die Kreatininkonzentration bezogen. Unter der Voraussetzung einer einigermaßen konstanten täglichen Kreatininausscheidung innerhalb bestimmter Altersgruppen ist die tägliche Aminosäurenausscheidung proportional dem Quotienten aus Aminosäuren- und Kreatininkonzentration. 


\section{Pfortaderhochdruck}

\section{und}

\section{Eiweißstoffwechsel}

Indikation und metabolische Konsequenzen porto-kavalèr Anastomosen bei Leberzirrhosekranken

Von Priv.-Doz. Dr. Gregor Esser

Groß-Oktav. Mit 49 Abbildungen. VIII, 180 Seiten. 1969. Plastik flexibel DM 36,-

Das vorliegende Buch ist für die Zukunft besonders wertvoll, da die Krankheiten der Leber mit der Hepatitis an der Spitze ihrer Infektionskrankheiten in allen Ländern zahlenmäßig ansteigen und daher mit einer $\mathrm{Zu}$ nahme der ,jetzt schon häufigen Leberzirrhose" und im Gefolge des damit zusammenhängenden Pfortaderhochdrucks mit vermehrt auftretenden lebensbedrohlichen Varizenblutungen zu rechnen ist.

Das gut ausgestattete Buch enthält 49 Abbildungen und 56 Tabellen. Der Inhalt gliedert sich einschließlich der Einführung und Zusammenfassung in 9 Hauptkapitel. Angeschlossen sind eine ausführliche Literaturaufstellung und ein Sachverzeichnis.

Das Buch besitzt nicht nur einen speziellen Wert für die Klinik der Lebererkrankungen. Vielmehr ist es bei der heutigen zahlenmäßigen Verbreitung der Leberzirrhose für jeden Arzt von besonderer Wichtigkeit, den Inhalt dieser Arbeit zu kennen.

Rundscbreiben des Verbandes der für Berufsgenossenschaften tätigen Ärzte

Es werden die für die Indikation erforderlichen klinischen Untersuchungsmethoden eingehend geschildert sowie die Ergebnisse tierexperimenteller Forschungen, die mit den an Menschen gefundenen nicht immer übereinstimmen.

Die operative Kontraindikationen werden genau erörtert.

Prof. Dr. Franke in Mediziniscbe Neuerscheinungen

Walter de Gruyter \& Co $\cdot$ Berlin

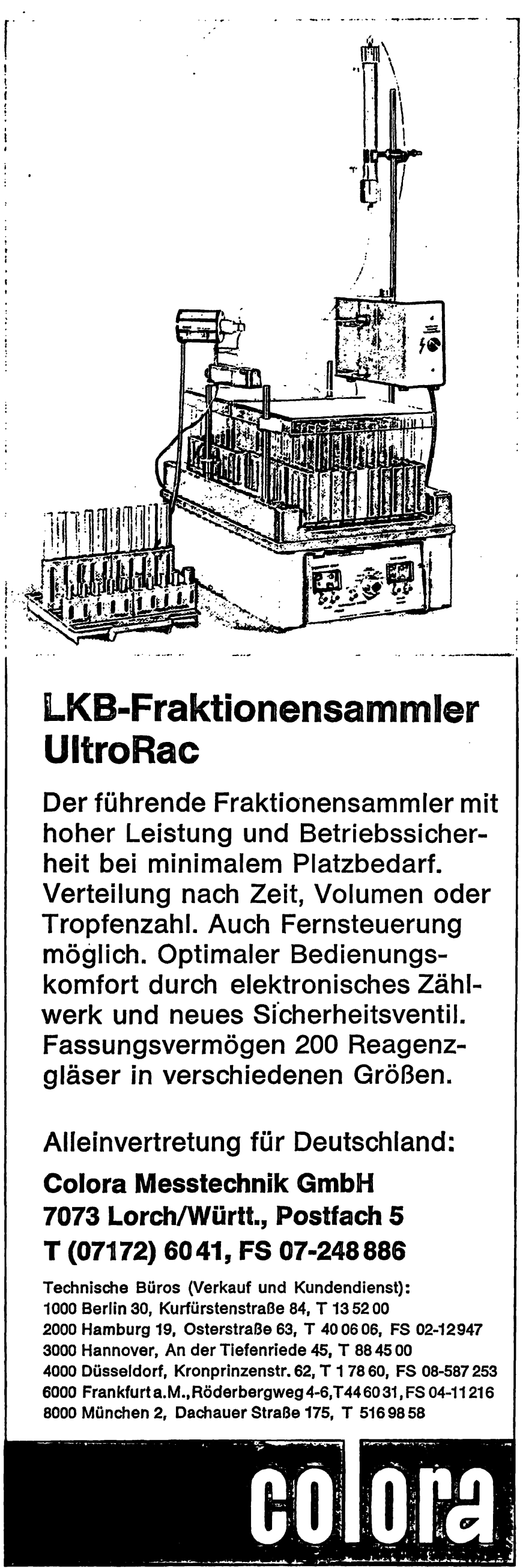




\section{BULLETIN \\ DE LA \\ SOCIÉTÉ DE CHIMIE BIOLOGIQUE}

(„Berichte der Gesellschaft für biologische Chemie“),

Unter Mitwirkung des

"CENTRE NATIONAL DE LA RECHERCHE SCIENTIFIQUE“

(National-Centrum für wissenschaftliche Forschung) veröffentlicht

J.-P. EBEL Secrétaire Général

(Relations

extérieures)
R. PERLES

Secrétaire Général
F. PERCHERON

Rédacteur en Chef Adjoint
Y. RAOUI

Rédacteur en Chef

\section{F. GROS}

Rédacteur en Chef Adjoint

Sekretariat und Redaktion: 4, avenue de l'Observatoire, Paris (6e)

Herausgeber: MAsson et $\mathrm{CIE}_{\mathrm{I}}$ 120, Boulevard Saint-Germain, Paris (6e)

Der „Bulletin de la Soctété de Chime Brologique“ veröffentlicht jährlich 11 Hefte; diese enthalten die Arbeiten der französischen Biochemiker, welche der „SOCIÉTÉ DE CHIMIE BrologrQue“ (Gesellschaft für biologische Chemie) angehören.

Abonnementspreis 1969:

Frankreich und „Franc-Zone“ . . $150 \mathrm{ffrcs}$

Belgien . . . . . . . . . 1684 bfres

Andere Länder . . . . . . . 165 ffres

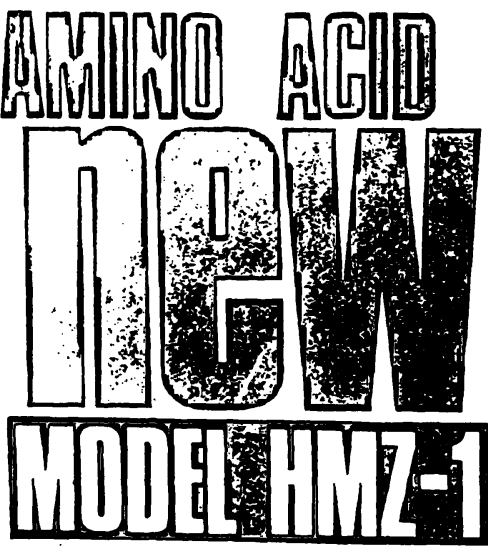

Semi Mikro Attachment

Automatic Analyses on

Picomol - Samples

Sensitivity: 20 Picomol
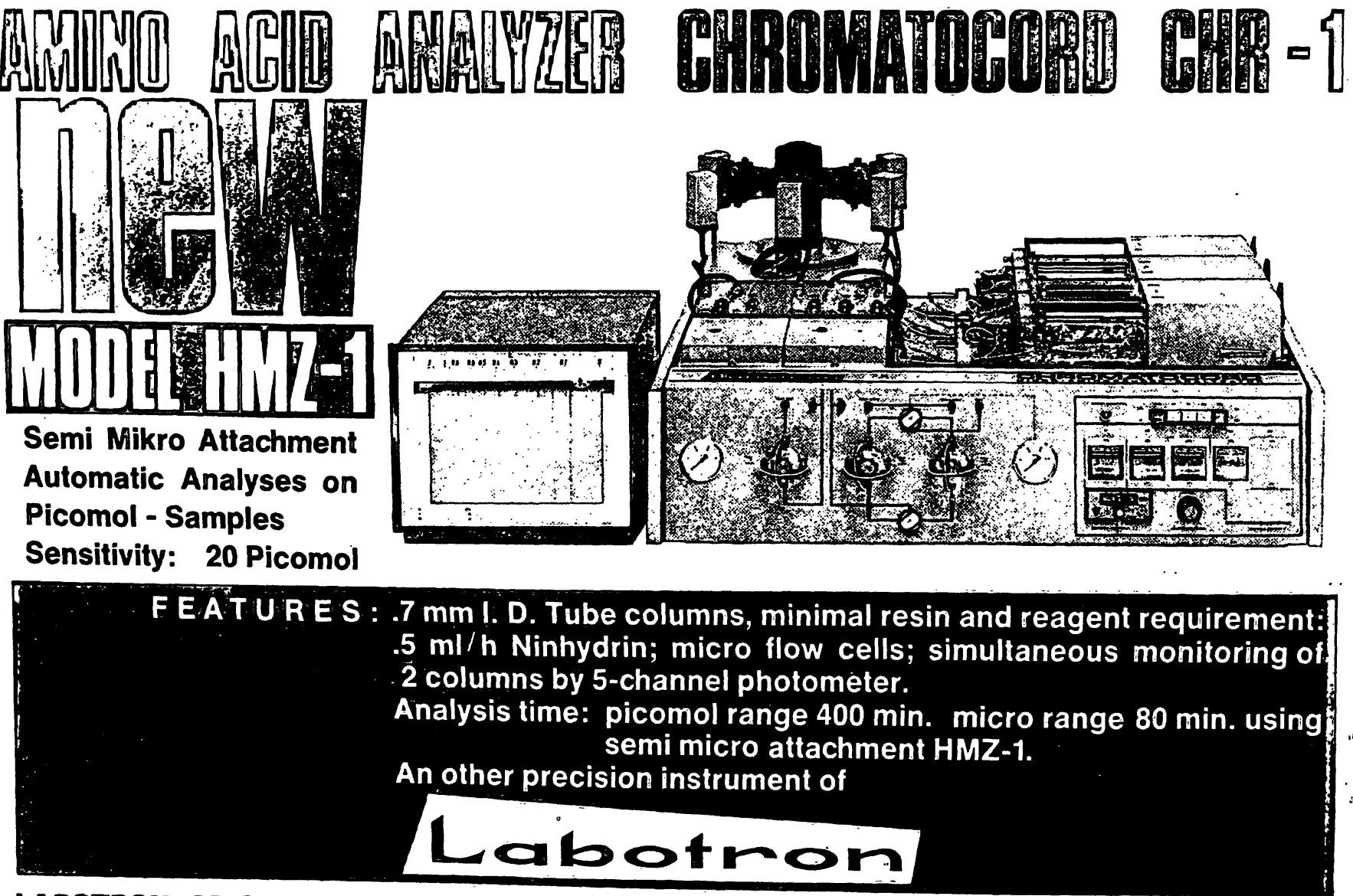

2 columns by 5 -channel photometer.

Analysis time: picomol range $\mathbf{4 0 0} \mathrm{min}$. micro range $\mathbf{8 0} \mathrm{min}$. using

An other precision instrument of 
Kreatinin wurde mit Hilfe der bekannten und auch in Automaten erprobten Pikrinsäuremethode nach FoLIN-Wu ermittelt (Methode Technicon Nr. 11a).

\section{Manuelle Bestimmungsmethode}

Reagenzien

1. Phosphatpuffer, $0,25 \mathrm{M}, \mathrm{pH} 7,0$.

2. 5,5'-Dithio-bis-(2-nitrobenzoesäure), $4 \mathrm{~mm}$ in 0,25M Phosphatpuffer, $\mathrm{pH} 7,0$.

3. Natriumcyanid-Lösung, $1 \mathrm{M}$ in dest. Wasser. Die Lösung wurde jeden Tag neu angesetzt.

4. Cysteinstandardlösungen, $0,25,0,5,1$ und $2 \mathrm{~mm}$ Cystein in $0,05 \mathrm{~N} \mathrm{NaOH}$.

\section{Vorgehen}

\section{Herstellung der Eichleurve}

$0,4 \mathrm{~m} l$ dest. Wasser (Leerwert) und $0,4 \mathrm{~m} l$ der Standardlösungen werden jeweils mit $0,2 \mathrm{~m} l \mathrm{NaCN}$-Lösung versetzt. Nach 10 Min. werden zu jeder Probe $2,0 \mathrm{ml}$ Phosphatpuffer und $0,5 \mathrm{ml} 5,5^{\prime}-$ Dithio-bis-(2-nitrobenzoesäure)-Lösung hinzugegeben und gemischt. Nach $3 \mathrm{Min}$. mißt man die Extinktion der Standardlösungen gegen den Leerwert bei $405 \mathrm{~nm}$ in einem Filterphotometer (Eppendorf). Aus den Werten der Extinktion und der Konzentration der einzelnen Standardlösungen wird eine Eichkurve angefertigt (Abb. 1).

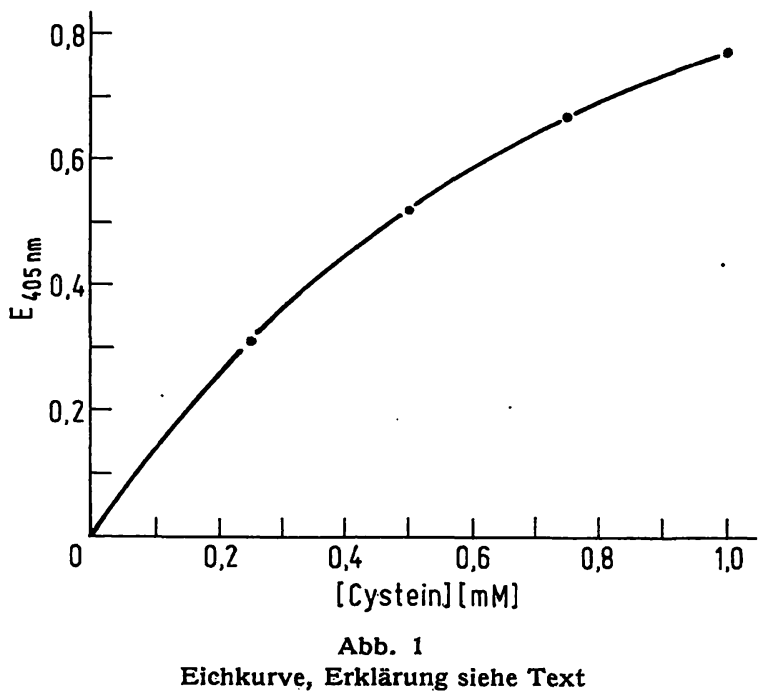

\section{Untersuchung der Urinprobe}

Zunächst wird der zu untersuchende Urin mit $0,1 \mathrm{~N} \mathrm{NaOH}$ im Verhältnis 1:1 verdünnt. Einen möglicherweise auftretenden Niederschlag läßt man sedimentieren oder man zentrifugiert bis zur Bodensatzbildung. Der Uberstand wird folgendermaßen weiterverwendet:

1. Ermittlung der Extinktion der Eigenfarbe des Ürins: $\mathrm{Zu}$ $0,4 \mathrm{ml}$ dest. Wasser (Leerwert) bzw. Ủberstand werden $0,2 \mathrm{ml}$ $\mathrm{NaCN}$-Lösung hinzugefügt. Nach $10 \mathrm{Min}$. gibt man $2,5 \mathrm{ml}$ Phosphatpuffer jeweils hinzu und mißt die Extinktion des Uberstandes gegen den Leerwert bei $405 \mathrm{~nm}$.

2. Bestimmung der Extinktion der SH-Gruppen des Urins: $\mathrm{Zu} 0,4 \mathrm{ml}$ dest. Wasser (Leerwert) bzw. Uberstand gibt man $0,2 \mathrm{ml} \mathrm{NaCN}$-Iösung, mischt und läßt die Ansätze $10 \mathrm{Min}$. stehen. Danach wird jeweils $2,0 \mathrm{~m} l$ Phosphatpuffer und $0,5 \mathrm{~m} l$ 5,5'-Dithio-bis-(2-nitrobenzoesäure)-Lösung hinzugefügt und gemischt. Nach 3 Min. wird die Extinktion der Urinprobe gegen den Leerwert bei $405 \mathrm{~nm}$ gemessen.

Unter Berücksichtigung der Extinktion der Urineigenfarbe kann die SH-Gruppenkonzentration in $\mathrm{mMol} / \mathrm{l}$ Urin der Eichkurve entnommen werden. $\mathrm{D}_{2}$ der Urin vor der Untersuchung im Verhältnis $1: 1$ verdünnt wurde, ist die der Eichkurve entnommene Konzentration noch zu verdoppeln.
Die Untersuchung der Abhängigkeit der gemessenen Extinktion von dex Zeit ergibt bei Cystein, Cystin und Homocystin, daß das Maximum jeweils nach $3 \mathrm{Min}$. erreicht ist.

Die Absorptionsspektren der Derivate dieser drei Aminosäuren gibt die Kurve I in Abbildung 2 wieder, sie weichen bei gleicher

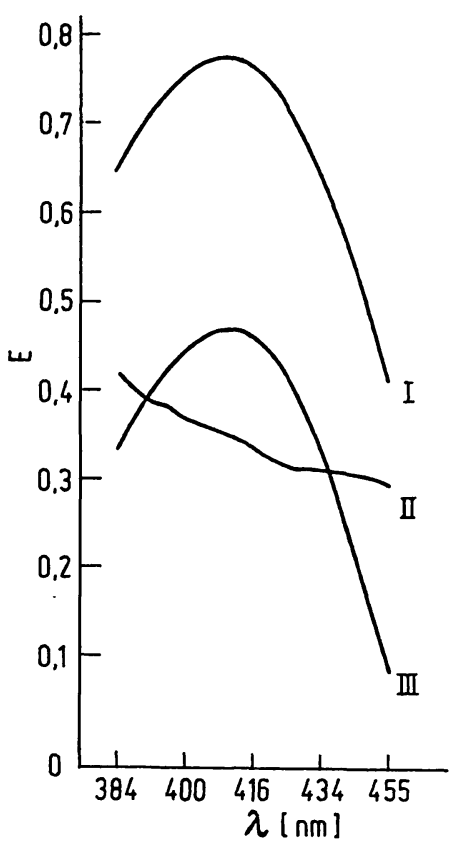

Abb. 2

Absorptionsspektren von 5,5'-Dithio-bis-(2-nitrobenzoesäure) mit Cystein, Cystin oder Homocystin (I), eines Urines mit starker EigenCystein, Cystin oder Homocystin (I), eines Urines mit starker Eigen-
farbe (II) und einer 5,5'-Dithio-bis-(2-nitrobenzoesäure)-Lösung, der eine NaCN-Lösung zugesetzt wurde

Konzentration der Lösungen nur unwesentlich in der Höhe der Extinktion voneinander ab. Ebenso liegt das Maximum ihrer Extinktion bei der gleichen Wellenlänge $(412 \mathrm{~nm})$, was nach der oben angegebenen Formelgleichung zu erwarten ist; denn in jedem Falle ist dic Freisetzung der 2-Nitro-5-thiobenzoesäureAnionen entscheidend.

Kurve II in Abbildung 2 gibt das typische Absorptionsspektrum eines Urines wieder, der eine sehr starke Eigenfärbung aufweist. Gibt man NaCN-Lösung zur 5,5'-Dithio-bis-(2-nitrobenzoesäure)Lösung, so wird eine bestimmte Menge 2-Nitro-5-thiobenzoesäure-Anionen freigesetzt. Das Absorptionsspektrum einer solchen Mischung ist die Kurve III in Abbildung 2. Ist eine bestimmte $\mathrm{NaCN}-$ Konzentration in der Mischung erreicht, so ist ihre Extinktion bei gleicher 5,5'-Dithio-bis-(2-nitrobenzoesäure)-Konzentration unabhängig von der Menge der zugesetzten $\mathrm{NaCN}-L$ ösung, die Extinktion bleibt gleich.

\section{Halbautomatische Bestimmungsmethode}

Verwendet wurde das Auto-Analyzer-System der Firma Technicon, Ardsley, New York.

Den schematischen Aufbau zeigt die Abbildung 3.

Die Reagenzien sind die gleichen, wie oben beschrieben. 40 Proben pro Stunde werden aus einem Probengeber mittels einer Proportionspumpe angesaugt und mit $\mathrm{NaCN}-L$ ösung gemischt. Gleichzeitig wird die Probe durch Stickstoff segmentiert. Wird Luft benutzt, so besteht die Gefahr der Oxydation der freigesetzten SH-Gruppen. Nach $10 \mathrm{Min}$. wird die Pufferlösung zugemischt, kurz darauf die 5,5'-Dithio-bis-(2-nitrobenzoesäure)Lösung und schließlich nach $3 \mathrm{Min}$. die Extinktion der Probe in einem Durchfluß-Kolorimeter (Filter $410 \mathrm{~nm}$, Schichtdicke $1,5 \mathrm{~cm}$ ) gemessen. Ein Schreiber zeichnet die gemessenen Extinktionswerte fortlaufend auf (siche Abbildung 4).

$\mathrm{Da}$ die Grundextinktion bei dieser Methode schon relativ groß ist, reichte die im Durchfluß-Kolorimetcr eingebaute Abgleichung zur Einstellung eines Null-Wertes nicht aus. Es mußten zusätzlich ein Potentiometer zwischen Meß- und Referenzphotozelle des Kolorimeters sowie ein Graufilter vor die Referenzphotozelle 


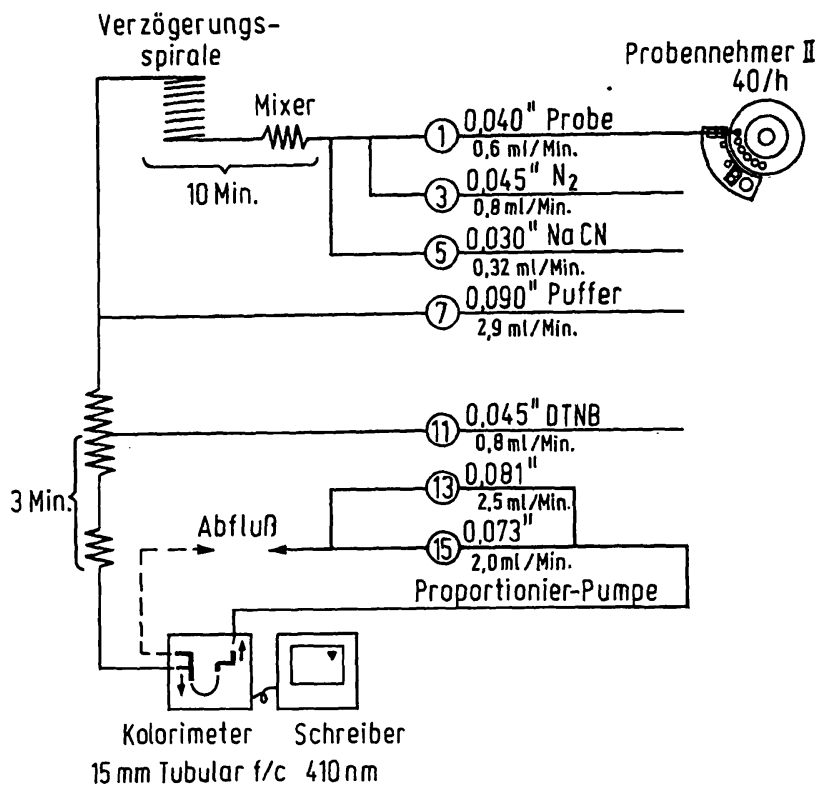

Abb. 3

Schema der Apparatur zur halbautomatischen Bestimmung. Erklärung siehe Text

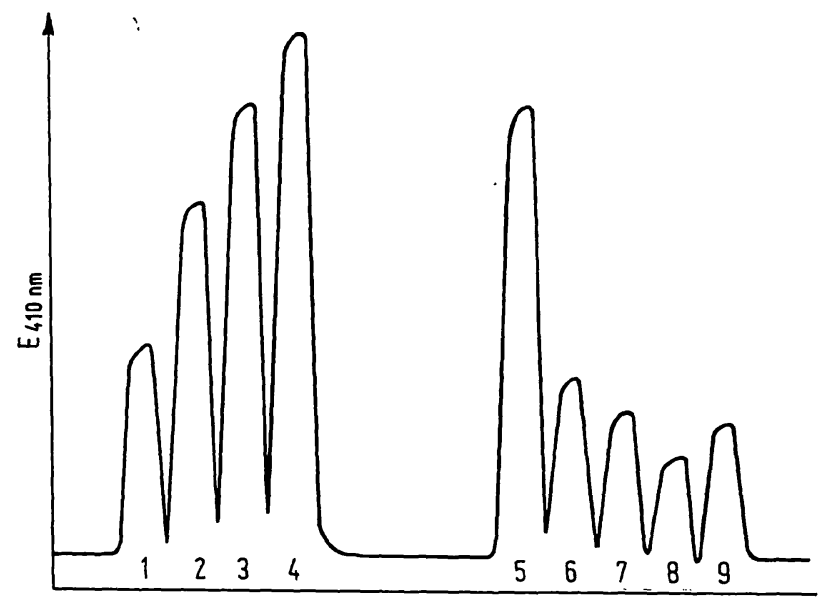

Abb. 4

Fortlaufend mittels eines Linearschreibers registrierte Extinktion von Cystein-Standard-Lösungen, des Urins eines an Cystinurie erkrankten
Kindes und von normalen Urinen

gesetzt werden. Die Höhen der ersten 4 dargestellten Zacken in Abbildung 4 entsprechen den Extinktionen der Standardlösungen mit 0,25, 0,5, 1 und $2 \mathrm{~mm}$ Cystein in dest. Wasser. Mit Hilfe der Extinktions- und Konzentrationswerte der Standardlösungen wird eine Eichkurve angefertigt (Abb. 1). Die Peaks 6-9 in Abbildung 4 entsprechen den Extinktionen von normalen Utinen, bei Peak 5 lag eine Cystinurie vor.

Bei den letzten Zacken in dieser Abbildung sind die Extinktionen der Eigenfarbe der Urine noch nicht berücksichtigt. Dazu werden die Extinktionen der Proben nach Ersatz der 5,5'-Dithio-bis(2-nitrobenzoesäure)-Lösung durch Pufferlösung ein zweites Mal gegen einen Leerwert, bei dem die vorgegebene Urinmenge durch die gleiche Menge an dest. Wasser ersetzt wird, bei sonst gleichen Bedingungen gemessen. Die Extinktionen der freigesetzten SH-Gruppen ergeben sich aus den Differenzen der beiden aufgezeichneten Zacken. Auf der Eichkurve können die Konzentrationen der SH-Gruppen in $\mathrm{mMol} / l$, die dieser Extinktion entspricht, abgelesen werden.

\section{Ergebnisse und Diskussion}

Die Urine von 593 unausgewählten Patienten unserer Klinik im Alter von 1 Tag bis zu 17 Jahren wurden mit dem beschriebenen automatischen System untersucht, außerdem Urine von an Cystinurie und Homocystinurie erkrankten Kindern und Jugendlichen. Die gemessenen Konzentrationen der SH-Gruppen (pro S-S-Brücke wird. nur eine SH-Gruppe freigesetzt) wurde auf die Kreatinin-Konzentration bèzogen.

Die Errechnung der Normalwerte mit seinen Spannweiten erfolgtẻ folgendermaßen: $10 \%$ der Werte wurden als relativ erhöht angesehen. Die übrigen Werte wurden in Gruppen, entsprechend einer Kreatinin-Konzentrationsspanne von 10 oder $20 \mathrm{mg} / 100 \mathrm{ml}$, zusammengefaßt. Innerhalb einer solchen Gruppe wurde der Medianwert der SH-Gruppenkonzentration errechnet (in den folgenden Abbildungen als Kreise gezeichnet). Der Abstand von dem höchsten bzw. dem niedrigsten Wert dieser Gruppe gibt die Spannweite an (als ausgezogene Linien dargestellt).

Bei den Untersuchungen stellte sich heraus, daß die Medianwerte und Spannweiten der Konzentrationen in Urinen von Kindern verschiedenen Alters nicht übereinstimmten. Es lassen sich folgende Gruppen aufstellen:

1. Frühgeborene

2. Reifgeborene bis 2 Jahre alte Kinder

3. 2-6 Jahre und

4. 6-17 Jahre

Vergleicht man die Verbindungslinien der Medianwerte in den Abbildungen 6 bis 8, so erkennt man, daß mit fortschreitendem Alter ihre Steigung abnimmt. Die SH-Gruppen-Konzentration vergrößert sich also nicht in dem Maße wie die Kreatininkonzentration. Bei Untersuchungen von Fällen von Cystinurie und Homocystinurie stellt sich aber die Frage, ob die SH-Gruppenkonzentration nicht so stark vom Normalkollektiv abweicht (Homocystinurie Nr. 9 in Abb. 7, Cystinurie Nr. 8, 9, 10 und 11 in Abb. 8), daß die altersabhängigen Unterschiede der SH-Gruppenkonzentrationen, bezogen auf Kreatinin-Konzentrationen, vernachlässigt werden können. Bei der hier angewandten Methode zur Feststellung von Normalwertbereichen fallen eine ganze Reihe von Testen positiv aus, bei denen es sich um generalisierte Hyperaminoacidurien handelt. Es sind dies

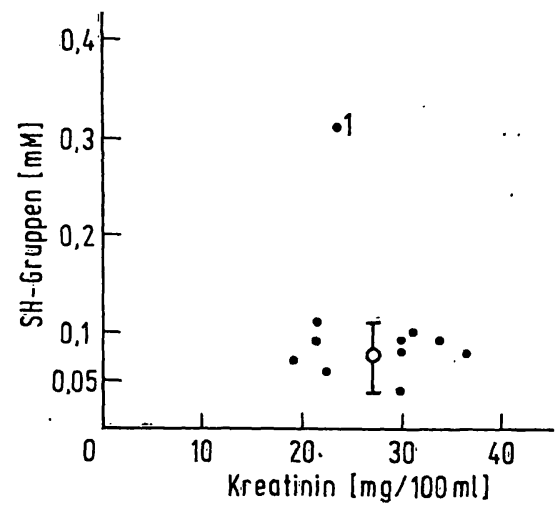

Abb. 5

SH-Gruppen- und Kreatinin-Konzentrationen in Urinen von Frühgeborenen 

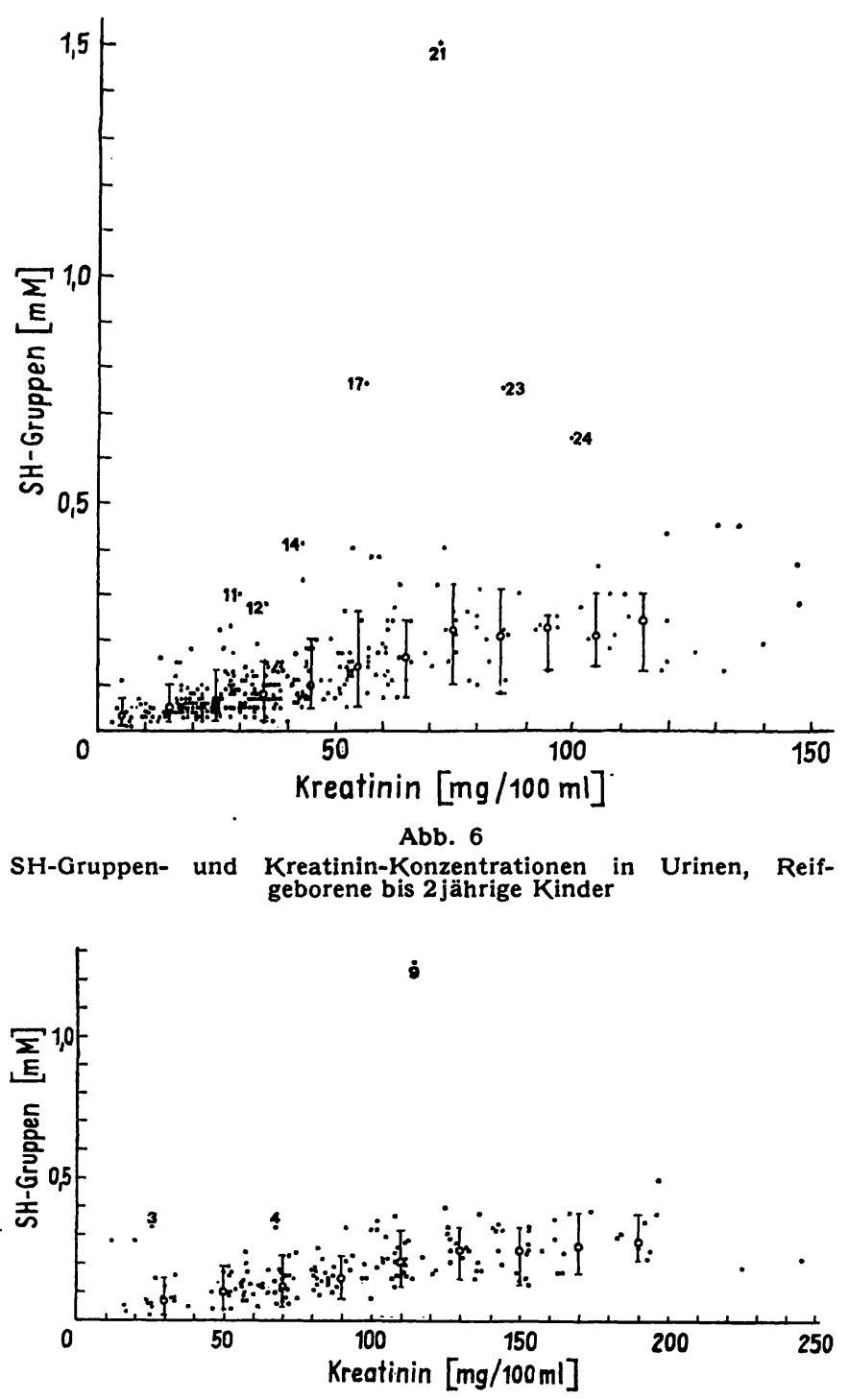

Abb. 7

SH-Gruppen- u. Kreatinin-Konzentrationen in Urinen von 2- bis 6 jährigen Kindern. Bei Nr. 9 lag eine Họmocystinurie vor

beispielsweise Rachitiden (Nr. 21 in Abb. 6, Nr. 4 in Abb. 7 und Nr. 4 in Abb. 8), Nephrosen (Nr. 3 in Abb. 7 und Nr. 1 und 7 in Abb. 8), Atemnotsyndrome bzw. Asphyxie (Nr. 12, 14, 17 in Abb. 6), Dyspepsie und azetonämisches Erbrechen (Nr. 11 in Abb. 6 und Nr. 6 in Abb. 8), Ileus (Nr. 24 in Abb. 6) und Duodenalstenose (Nr. 23 in Abb. 6). Die bei den Frühgeborenen

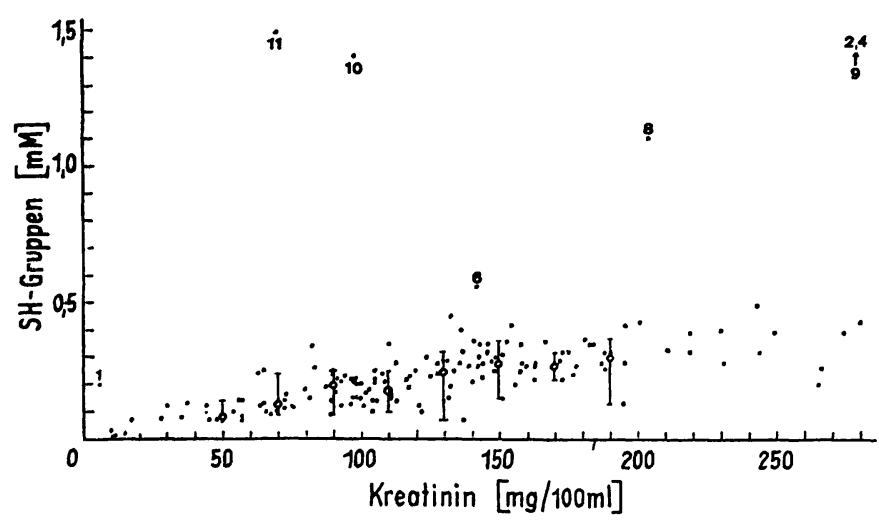

Abb. 8

SH-Gruppen- und Kreatinin-Konzentrationen in Urinen von 6- bis 17 jährigen Kindern und Jugendlichen. Bei 8, 9, 10 und 11 lag eine Cystinurie vor

gefundenen Werte zeigen deutlich die Konzentrierunfähigkeit dieser Nieren, so $\mathrm{da}$ B nicht eine Linie als Abhängigkeit von SH-Gruppen- zu Kreatinin-Konzentration im Urin zu finden ist, sondern lediglich eine Punktwolke. Bei dem einzigen aus diesem Bereich herausfallenden Punkt (Nr. 1 in Abb. 5) handelt es sich um eine generalisierte Hyperaminoacidurie bisher ungeklärter Ursache.

Bei der Kontrolle der Beeinflussung der 5,5'Dithio-bis(2-Nitrobenzoesäure)-Methode durch höhermolekulare Substanzen (Proteine) ergaben sich bei 40 Urinen vor und nach Enteiweißung mit Trichloressigsäure Differenzen, die weit unterhalb einer 10\%-Grenze lagen. Bei einer Nephrose mit Eiweißausscheidungen von ungefähr 10\% Esbach lag die Differenz bei $20 \%$. Im Hinblick auf die Anwendung der 5,5'Dithio-bis-(2-Nitrobenzoesäure)-Methode als Screening-Test auf Cystinurie und Homocystinurie spielt diese zusätzliche Erhöhung keine Rolle, da bei den gesuchten Krankheiten die SHGruppenkonzentration stark erhöht ist und die Krankheitsbilder sich ohnehin leicht voneinander abgrenzen lassen.

Werden Substanzen mit SH-Gruppen bzw. S-S-Bindungen im Urin vermehrt gefunden, so muß eine anschließende chromatographische Auftrennung der Aminosäuren klären, um welche es sich hierbei handelt.

Die untersuchten Homocystin- und Cystinurien zeigen, $\mathrm{da}$ sich der beschriebene Test gut zur Suche nach diesen Erkrankungen eignet.

\section{Literatur}

1. Ellman, G. L., Arch. Biochem. Biophysics 82, 70 (1959). - 2. Sullivan, M. X., W. C. Hess und H. W. Howard, J. biol. Chemistry 145, 621-624 (1942):

Dr. E. Mönch

Universitäts-Kinderklinik

1 Berlin 19

Heubnerweg 6 